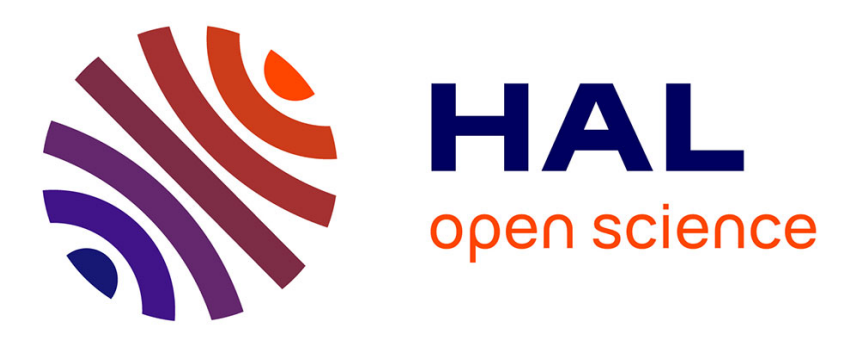

\title{
Misorientation dependence grain boundary complexions in symmetric tilt Al grain boundaries
}

Prakash Parajuli, David Romeu, Viwanou Hounkpati, Rubén Mendoza-Cruz, Jun Chen, Miguel José Yacamán, Jacob Flowers, Arturo Ponce

\section{- To cite this version:}

Prakash Parajuli, David Romeu, Viwanou Hounkpati, Rubén Mendoza-Cruz, Jun Chen, et al.. Misorientation dependence grain boundary complexions in symmetric tilt Al grain boundaries. Acta Materialia, 2019, 181, pp.216-227. 10.1016/j.actamat.2019.09.010 . hal-02334094

\section{HAL Id: hal-02334094 \\ https://hal.science/hal-02334094}

Submitted on 28 Oct 2019

HAL is a multi-disciplinary open access archive for the deposit and dissemination of scientific research documents, whether they are published or not. The documents may come from teaching and research institutions in France or abroad, or from public or private research centers.
L'archive ouverte pluridisciplinaire HAL, est destinée au dépôt et à la diffusion de documents scientifiques de niveau recherche, publiés ou non, émanant des établissements d'enseignement et de recherche français ou étrangers, des laboratoires publics ou privés. 


\title{
Misorientation dependence grain boundary complexions in $<111>$ symmetric tilt Al grain boundaries
}

\author{
Prakash Parajuli a , David Romeu ${ }^{\mathrm{b}}$, Viwanou Hounkpati ${ }^{\mathrm{c}}$, Rubén Mendoza-Cruz ${ }^{\mathrm{a}}$, \\ Jun Chen ${ }^{c}$, Miguel José Yacamán a , Jacob Flowers a, Arturo Ponce ${ }^{\mathrm{a}, *}$ \\ a Department of Physics and Astronomy, The University of Texas at San Antonio, One UTSA Circle, San Antonio, TX, 78249, USA \\ ${ }^{\mathrm{b}}$ Instituto de Física, Universidad Nacional Autónoma de México, Apartado Postal 20-364, 01000, Mexico City, Mexico \\ ${ }^{\mathrm{c}}$ Normandie Univ, ENSICAEN, UNICAEN, CEA, CNRS, CIMAP, 14000, Caen, France
}

\begin{abstract}
Since polycrystalline alloys consist of a complex network of various types of grain boundaries (GBs), detailed atomic-scale analysis of how some impurities are distributed at every type of GBs is necessary to fully understand the implications of GB segregation on material's performance. In this study, we present the atomic-scale structural combined with a chemical analysis of segregation induced GB complexions across the various types of $\mathrm{Al}$ alloy $7075 \mathrm{GBs}$ using aberration-corrected microscopy and crystal orientation mapping assisted with precession electron diffraction. The result shows multilayer Cu GB segregation containing non-uniformly segregated mixed atomic columns across the interfaces. Two distinct types of Cu GB segregation behavior were observed, point and parallel array, analyzed by means of a displacement field obtained from the dichromatic pattern. Atomistic simulations were performed to test the energetic feasibility of the observed segregation behavior. As per the knowledge of the authors, this is the first report on experimental analysis of segregation induced periodic ordered structured GB complexions on $\mathrm{Al}$ alloy system. Furthermore, every GBs of the films were segregated uniquely forming ordered structures along the interface. The distance between two consecutive high segregated units was periodic for the point segregated GBs and followed a trend of a theoretical model of dislocation spacing. Based on the distance between two high segregated units, it is inferred that highly misorientated GBs are more segregated than low misoriented GBs. This study demonstrates that the misorientation between the neighboring grains significantly influences the segregation behavior across the interface and consequently, the structure of segregation-induced GB complexions.
\end{abstract}

\section{Introduction}

Most of the materials used in technological applications are polycrystalline: i.e., they consist of a substantial number of regularly arranged atomic units (grains) with characteristics orientation, and interfaces where two grains of the same phase differing in mutual orientations meet [grain boundaries(GBs)] [1,2]. Since GBs forms a transition region between the neighboring grains, the lattice mismatch at those regions causes local structural disorder and an excess energy configuration. This high energetic configuration promotes the diffusion of atomic defects, impurities and alloying elements in a multicomponent alloy to the GBs leading to their

\footnotetext{
* Corresponding author.

E-mail address: arturo.ponce@utsa.edu (A. Ponce).
}

preferential accumulation $[3,4]$ resulting in the drastic local chemical composition alteration at the GBs [5]. Nowadays, various multicomponent alloys are designed to improve the desired property of the material. However, in some cases, the properties of the materials diminish catastrophically due to the preferential accumulation of the alloying elements at the GBs, causing decohesion and GB embrittlement $[2,4,6]$. Understanding how and why these effects occur at the atomic scale by resolving the sites and chemical identities of the parent and alien atoms comprising the interface is a crucial step towards engineering beneficial materials that can resist such deleterious effects.

Recent advances in characterization techniques for the study of interfaces have uncovered important aspects of structuredependent GB properties [7] and the GB segregation phenomena [6] that impacts the macroscopic behavior of the alloyed materials. It has been well perceived in interface research that the GB 
segregation can be used as a microstructure design method since accumulating solutes affect the structure, phase state, and atomic bonds within the decorated interface. More specifically, segregation of certain solutes can strengthen or weaken the interface by GB cohesion or decohesion and might result in phase transformation of or at the GB region (GB Complexions [8]) [2,9]. Despite its enormous importance, published reports on atomic scale characterization and analysis of GB segregation and GB complexions are largely inadequate. The reason for this could be the immense experimental challenges associated with its quantitative characterization and also the anisotropies in GB character [10], energy [11] and segregation [12]. A handful of studies have examined the periodic adsorbate pattern at the GBs [13-22]. These studies are limited to the examination of the specific type of GBs (twin, $\sum 5$, and some $r$ HAGBs). Furthermore, formation of various types of segregation induced GB complexions [23-27], and the theoretical analysis on GB complexion transformation for HAGBs at high temperature has been reported [27,28].

At a macroscopic scale, a grain boundary is characterized by five degrees of freedom, three variables required to specify the rotation (axis and angle) describing the misorientation between the adjacent lattice crystals connected by the boundary, and the other two variables associated with specifying the normal to the boundary plane [29]. Among the five parameters, misorientation between two neighboring grains is one of the most important property of GB. This is a basis of classification of GBs on coincidence site lattice (CSL) model, a most commonly used model until now to classify GBs despite the fact that it suffers some limitations [30]. Several attempts are made to study GBs complexions along particular GBs (twin or $\sum 5$ ) or some randomly selected GBs. However, there are no comprehensive analyses of misorientation dependence on GB complexions structure. It is well established that the polycrystalline materials are comprised of different types of GBs and the materials bulk properties depend on the types of GBs, their structure and chemistry, and how they are connected in the material. Hence, a comprehensive atomic-scale study regarding how distinct types of GBs of multicomponent alloys are segregated is necessary to estimate the actual implications of GB segregation. Unfortunately, as per the knowledge of the author, the vast majority of the studies on GB segregation are concentrated on the segregation effects rather than atomic scale configuration of adsorbate at the GBs. To bridge this gap, an atomic-scale analysis of the GBs of a $<111>$ textured Al7075 alloy thin film was performed, demonstrating $\mathrm{Cu}$-segregation behavior along different GBs.

Herein, we report the atomic-level structure of $\mathrm{Cu}$ segregation induced GB complexions at the various GBs (LAGBs, special GBs, and random HAGBs) of $\mathrm{Al} 7075$ alloy showing distinct types of segregation behavior. Firstly, the important structural features such as grain size, texture, and boundary type and connectivity distribution into the films were examined by using automated crystal orientation mapping assisted with precession electron diffraction. Following the results, the atomic scale adsorbate arrangement patterns across the GBs were revealed through aberrationcorrected scanning transmission electron microscopy (STEM) imaging. Structural model of the grain boundaries is proposed as well as the segregation behavior was energetically studied by atomistic simulations. Detailed spectroscopy analyses (energy dispersive Xray spectroscopy (EDS) and electron energy loss spectroscopy (EELS)) were performed to identify the chemical nature of segregating element.

\section{Methods}

The Al alloy 7075 films examined in this study were grown by a physical vapor deposition technique $[21,31,32]$. Small pieces of a commercially available Al Alloy 7075 foil (chemical composition: Al $90 \%$, Zn 5.5\%, Mg 2.5\%, Cu 1.5\%, and Si $0.5 \%$ ) were thermally evaporated and deposited on freshly cleaved commercial (100) $\mathrm{NaCl}$ crystals in high vacuum $\left(10^{-5}\right.$ torr $)$. The thin films were prepared at a deposition temperature of $200^{\circ} \mathrm{C}$, and a rate of $\sim 10 \AA / \mathrm{min}$. The final thickness of the film was $\sim 400 \AA$. Following deposition, the sample was slowly cooled down to room temperature in vacuum. The substrate was dissolved in water to transfer the film to TEM grids for further characterization.

STEM images in high-angle annular dark field mode (HAADFSTEM), electron EDS spectra, and EELS spectra were registered in a $200 \mathrm{kV}$ - JEOL ARM 200F microscope equipped with a CEOS Csaberration corrector. Microstructural features (texture, grain boundary character, and connectivity distribution) of the films were examined by using electron diffraction-assisted automated crystal orientation mapping provided by ASTAR software package. In this technique, sets of electron diffraction patterns were obtained by scanning the region of interest by precessed electron beam (precession angle $0.6^{\circ}$ ); these patterns were stored and subsequently indexed and analyzed using an automatic crosscorrelation procedure with a database of theoretical templates corresponding to all the possible orientations and crystallographic structures [33].

To confirm experimental results, two different representative GBs (different in terms of GB structures and segregation sites) were investigated by atomistic simulations: $\sum 13 \mathrm{~b}\left(<111>/ 27^{\circ}\right)$ and $\sum 7$ $\left(<111>/ 38^{\circ}\right)$. The equilibrium $0 \mathrm{~K}$ structure and energy was calculated for each GB. The simulations were performed with the code, LAMMPS (Large-scale Atomic/Molecular Massively Parallel Simulator) [34]. Embedded atom method (EAM) potentials for Al [35] and $\mathrm{AlCu}$ [36] were used to generate the minimum energy GBs. These potentials have been well tested and demonstrated to give a good evaluation of a large set of experimental and first-principles data [35-37]. A bicrystal model [38-40] with three-dimensional (3D) periodic boundary conditions consisting of two grains is employed. This model is created by constructing two separate crystal lattices with different crystallographic orientation and joining them together along a given axis. Further details of the simulation methodology that was used to obtain the minimum energy grain boundary structures can be found in Ref. [38]. After the procedure of energy minimization, the energy of each equilibrium GB structure is then calculated and compared to find the possible global minimum energy configuration. The size of the bicrystal models is $60.7 \AA \times 41.2 \AA \times 20.2 \AA(\mathrm{X} \times \mathrm{Y} \times \mathrm{Z})$ and the total number of atoms in each model is approximately 3120 . This cell size was sufficient to study the stability of GB structures of $\Sigma 7$ and $\Sigma 13 \mathrm{~b}$ and its probable segregation sites. The visualization tool Ovito [41] is used to illustrate GB structures.

\section{Results}

Microstructural features of the prepared thin films namely, crystal orientation, and GB character and connectivity distribution are summarised in Fig. 1. Fig. 1(a,e) show representative orientation maps of a large sample area of the prepared thin film viewed along $\mathrm{Z}$ and Y-direction respectively, in which each color represents a crystallographic direction viewed from a particular axis zone following the color code (Fig. 1(d)). Here, the $Z$ direction refers to the view direction parallel to the electron beam, while Y-axis relies on the paper plane and provides complementary information about the orientation of the crystallites. Diffraction patterns obtained by the precessed electron beam from the area marked by 1 and 2 in the orientation maps are presented in Fig. 1(b) and (c) respectively. There is no evidence of overlapping diffraction patterns, indicating that the grains are continuous through the film thickness. The 

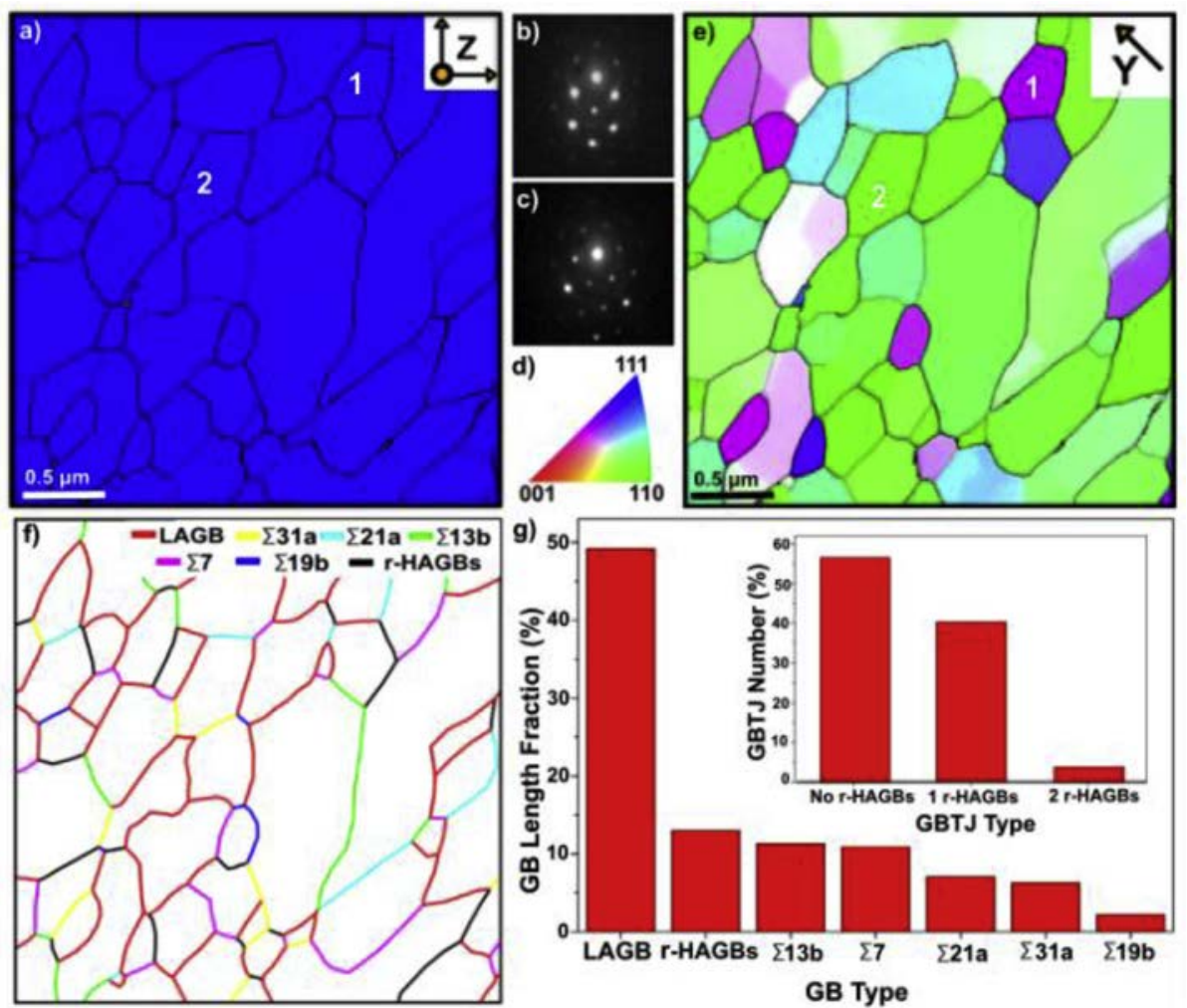

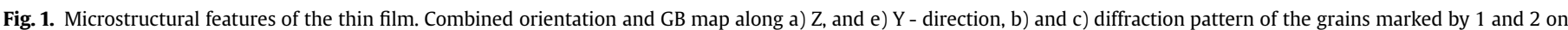

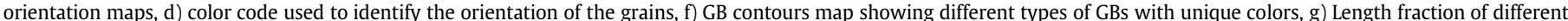

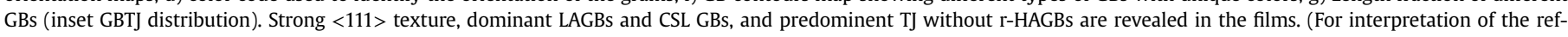
erences to color in this figure legend, the reader is referred to the Web version of this article.)

figures revealed a $<111>$ preferential growth direction of the film, as well as predominant $<110>$ orientations when they are viewed along the $\mathrm{Y}$ direction.

Similar to the prior studies on vacuum deposited metal thin films, this $<111>$ strong texture of the film can be correlated to the preferred orientation of the Al alloy crystallites corresponding to the lower surface energy planes $[21,31,42]$. Overview of the GB character, i.e., grain boundary type distribution (GB contours map showing different types of GBs by unique colors and the length fraction of those GBs) and GBs connectivity (GB triple junction (TJ) distribution) are summarised in Fig. 1(f)-(g) respectively. It should be noted that, CSL boundaries were assigned in view of the rotation axis $<111>$ and Deschamps's criteria for CSL boundaries [43], and various types of GBs (low angle GBs), random high angle GBs (HAGBs), $\sum 13 \mathrm{~b}\left(<111>/ 27.79^{\circ}\right), \sum 7\left(<111>/ 38.21^{\circ}\right), \sum 21 \mathrm{a}(<111>/$ $\left.21.78^{\circ}\right), \sum 31 \mathrm{a}\left(<111>/ 17.90^{\circ}\right)$, and $\left.\sum 19 \mathrm{~b}\left(<111>/ 46.80^{\circ}\right)\right)$ are marked by red, black, lime, magenta, cyan, yellow and blue colors respectively. The GB length fraction graph in Fig. 1(g)) shows a dominant LAGBs presence in the film (disorientation $<15^{\circ}$, marked as LAGBs) followed by $\sum 13 \mathrm{~b}$ and $\sum 7$ CSL GBs almost in equal fraction. Furthermore, GB connectivity distribution, the parameter controlling mechanical properties [44], is presented in the inset to Fig. 1(g). It shows a dominance of TJ without r-HAGBs and too low TJ containing two r-HAGBs, this seems reasonable considering the proportion of r-HAGBs in the film. The results of the current study, strong $<111>$ texture, and abundance CSL boundaries can be correlated to the tendency towards minimum energy configuration,e consistent with the various reports on vacuum deposited fcc metallic thin films $[21,31,45]$ except the population of twin boundaries $\left(\sum 3-<111>/ 60^{\circ}\right)$. This absence of twin boundaries in our films can be ascribed to the high stacking fault energy of Al [46], to the absence of any external stress to create those boundaries [47] and the effect of strong $<111>$ texture that could have restricted the formation of twin boundaries [48].

\subsection{Grain boundary segregation}

\subsubsection{Spectroscopic analysis}

Detailed EDS and EELS spectroscopy analyses were performed to identity of the segregating element at the boundaries. EDS spectroscopy from a large sample area and a specific GB region, as well as EELS analysis at and near to GB are presented in Figs. 2 and 3. Several EDS and EELS spectra were acquired from various regions of the thin film. However, only one set of representative spectroscopy data are presented herewith. The EDS overview of the film from a large sample area ( $\mathrm{Al} 96.77 \%$, Zn 0.15\%, Mg 1.47\%, Cu 1.13\%, Si 0.5\%) in Fig. 2(b) and the high contrast on HAADF images (Fig. 3(a)) suggested the segregation of the heavy elements: $\mathrm{Cu}$ or $\mathrm{Zn}$ towards the GBs. For further confirmation, line scan, and composition analysis were performed from the GB regions (Fig. $3 \mathrm{a}-\mathrm{c}$ ). As seen in the spectra, the presence of copper is evident. Furthermore, the line scan shows clear segregation of $\mathrm{Cu}$ along the GBs, illustrating the accretion of $\mathrm{Cu}$ and depletion of $\mathrm{Al}$ at the GB.

To further confirm the chemical identity of the high contrast atoms at GBs, we performed an EELS analysis. Fig. 3(d and e) show summed EELS spectra acquired from both the regions: GB core and $5 \mathrm{~nm}$ away from GB including the entire EELS energy range of all the alloyed elements. Spectra show a distinct peak of $\mathrm{Cu}$ at the GB core providing definitive evidence of strong segregation of copper into the GB. Besides apparent $\mathrm{Cu}$ peak at GB, shoulder like structure 

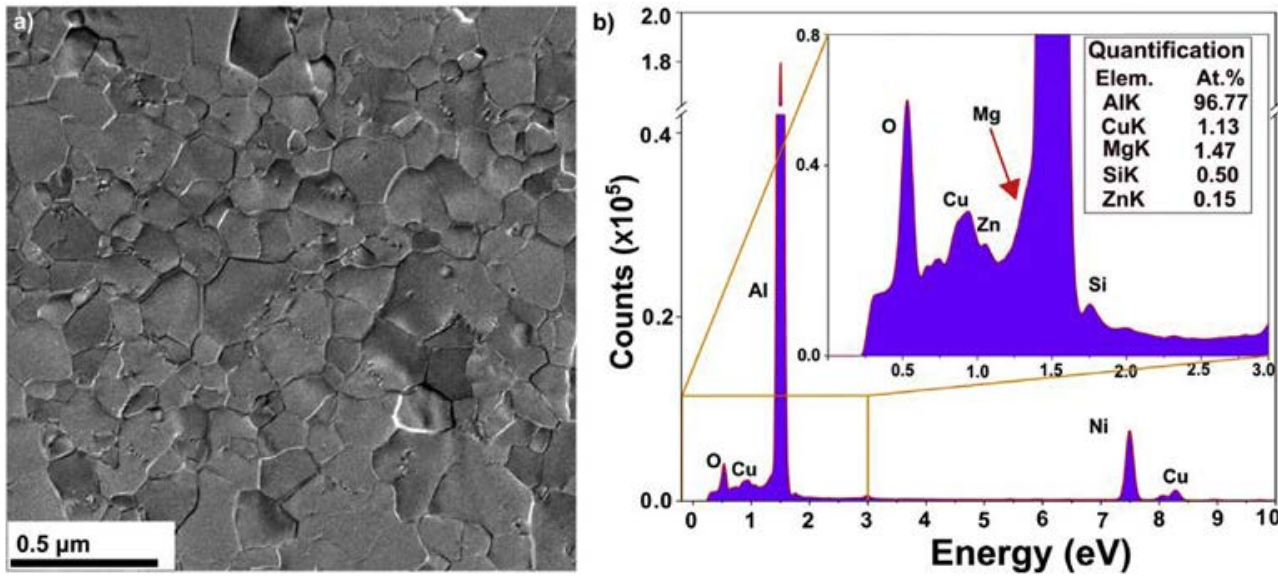

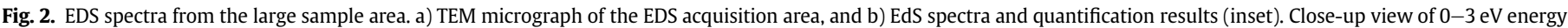
range are shown for the EDS spectra.

around $1035 \mathrm{eV}$ and a small peak around $1215 \mathrm{eV}$ can be seen in both the spectrum (GB core and away from the GB). These EELS energy ranges do not correspond to any of the alloying elements, and they appear on both spectrum. Hence we do not explore these in detail, as our concern was on the confirmation of segregating element at the boundary. Similar to the previous studies of this alloy (thin film prepared under another deposition conditions) [21], we encountered loss of stoichiometry for $\mathrm{Zn}$ in the film compared to the parent material ( $\mathrm{Al} \mathrm{90 \% ,} \mathrm{Zn} \mathrm{5.5 \% ,} \mathrm{Mg} \mathrm{2.5 \% ,} \mathrm{Cu} \mathrm{1.5 \% ,} \mathrm{Si} \mathrm{0.5 \% )}$ used to deposit film. Based on the Z-contrast principle of STEM HAADF imaging technique, and spectroscopic analysis (EDS (quantification, and line scan) and EELS), high contrast atoms segregated to the GB were confirmed as $\mathrm{Cu}$. Despite predominant segregation of copper, as a multicomponent alloy minor segregation (too low to be detected by our spectroscopic set up) of other alloying elements $\mathrm{Mg}$, Si, and $\mathrm{Zn}$ forming mixed atomic columns cannot be discarded. However, in this study, we were concentrated on the high contrast segregating element at the boundary. Cu GB segregation behavior at different types of GBs is described in details in the next section.

\subsubsection{Segregation behavior: an atomic scale analysis}

In order to explore the misorientation dependence on $\mathrm{Cu} G B$ segregation behavior, 25 different GBs of varying misorientation angles were analyzed in detail. Meticulous observation of the repeating segregating units in the HAADF images shows a series of various contrasting columns across the interface. Since the intensity of an atomic column in a STEM image is proportional to the atomic number of the elements present there [49], these contrast variation in different atomic columns along the boundary indicate a non-uniform level of segregation. Non-uniform level of segregation containing mixed atomic columns have been observed in GBs of multicomponent alloys [20,50]. It should be noted that a slightly brighter background of the GBs in the atom resolved HAADF images of some of the GBs appeared as an effect of diffused scattering of the electron beam at the GBs [51]. To get more insight on the varying intensity columns, intensity profiles of various columns across the different GBs were analyzed. Three categories of the intensity profiles were observed, first, brightest column/s, second, brighter columns (columns that are intense than grain matrix but less than that of brightest column), and finally, a grain matrix. Intensity profiles across two representative grain boundaries are shown in supporting information (Figure S1). Quantitively, brighter columns are $25 \%-50 \%$ intense than matrix whereas brightest columns are more than $100 \%$ intense. Referring to this varying intensity of atomic columns at the GB region, we used the following terminologies: high segregated column (brightest atomic column) and low segregated columns (columns brighter than matrix at the GB except the high segregated columns) throughout the paper to distinguish different high contrast columns of the GBs to make the explanation easy and clear. Furthermore, close examination of the segregation units across the various GBs showed two types of $\mathrm{Cu}$ multilayer (extended up to 4 atomic layers) GB segregation. First, Point segregation behavior, a high segregated column surrounded by other low segregated columns, and second, parallel array segregation behavior, two highly segregated columns opposite to each other across the interface surrounded by other low segregated columns. STEM-HAADF images of two representative GBs ( $\sum 13 \mathrm{~b}$ and $\sum 7$ ), close-up view of the segregating unit, and the model of pristine Al GB is shown in Fig. 4. The non-uniformly (high and low) segregated atomic columns across the interface showing point and parallel array GB segregation patterns are represented by different colored atoms.

Atomistic simulations were performed to test the energetically favorable segregation site for $\mathrm{Cu}$ along the GBs. Only high segregated columns were considered for energetic calculations. Driven by the experimental observation, pure atomic columns of pristine Al GB are replaced by mixed atomic columns (Al90Cu) across the various atomic sites: denoted as 1, 2, 3, 4 and 5 in Fig. 4. This concentration was taken considering approximate quantification results of point EDS analysis across the high segregated column. To support the study about the competition between these two types of segregation, both point and parallel array segregation pattern are considered for each GB: 3-point segregation $(1,2,3)$ and 2-parallel array segregation $(4,5)$ for $\sum 13 \mathrm{~b}$ (Fig. $4(\mathrm{c})$ ), and 1-point (3) and 2parallel $(1,2)$ for $\sum 7$ (Fig. 4(f)). GB energy after segregation and GB excess energy due to segregation corresponding to the segregated sites for $\sum 13 \mathrm{~b}$ and $\sum 7$ are plotted in Fig. 5(a)-b respectively.

Atomistic simulations yields GB energies that agree with the order of magnitude of existing calculated energies for Al GBs [38,52]. The GB structures associated with these energies also agree with the experimentally observed HAADF-STEM $\sum 7$ and $\sum 13 \mathrm{~b}$ GB structures (Fig. 4). Results show that for $\sum 13 \mathrm{~b}$ GB point segregation behavior is more energetically favored than a parallel array and vice-versa for $\sum 7 \mathrm{~GB}$. Furthermore, it can be seen in the figure that GB energy decreases irrespective of the segregation site. This result shows that the GB is a preferential segregation site for $\mathrm{Cu}$ atoms. To further explore the insights of the various segregation induced GB complexions, different types of the GBs based on their CSL classifications were analyzed individually. Representative STEM-HAADF 

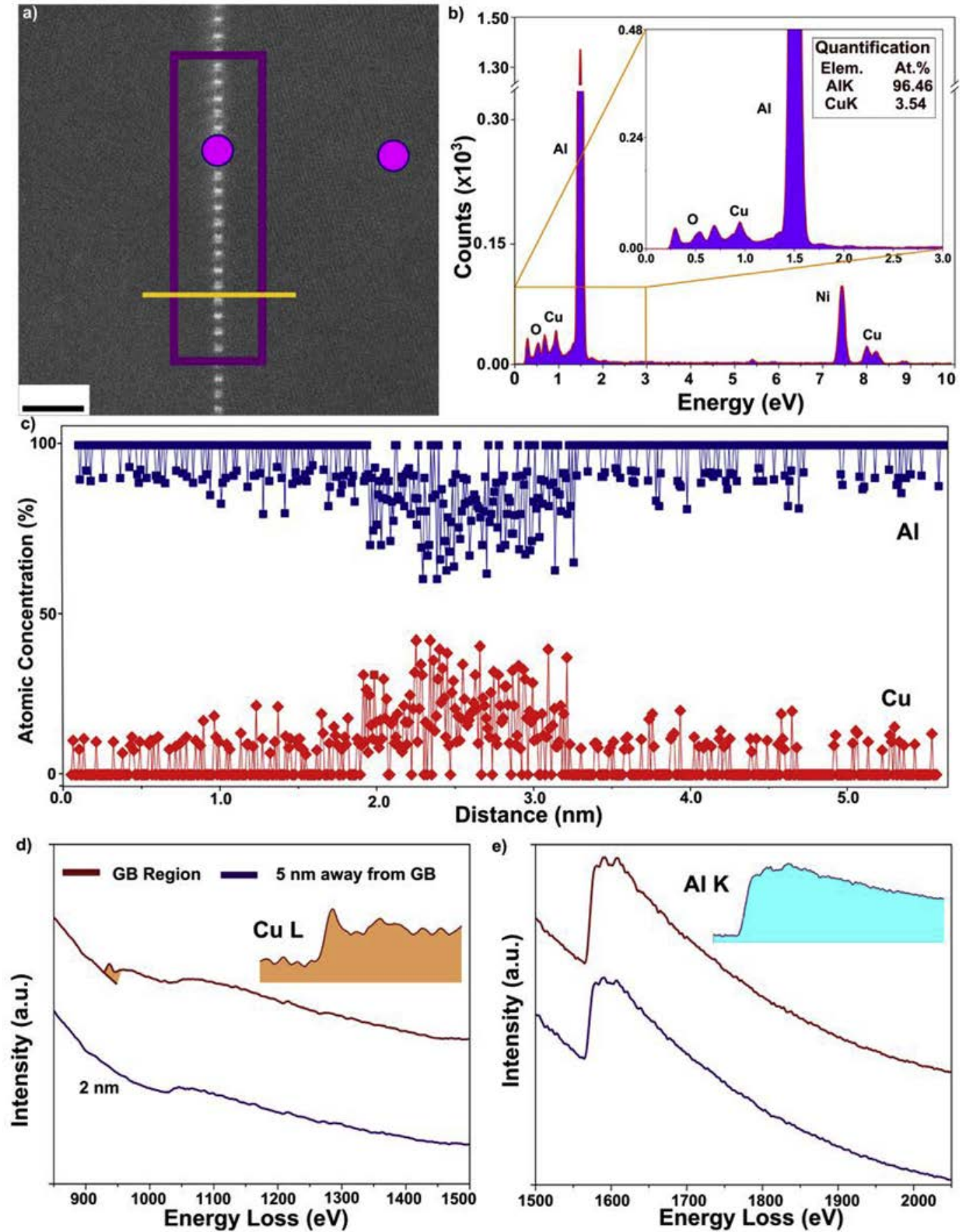

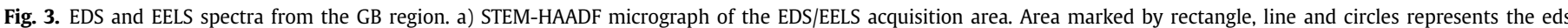

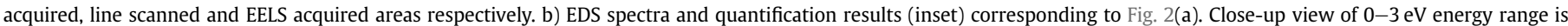

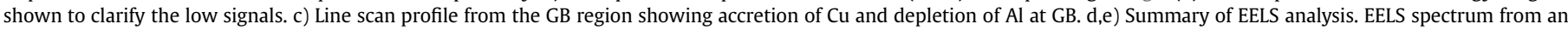

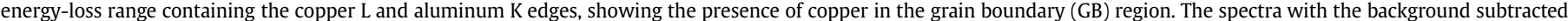
are shown at the top right of the corresponding Figures.

images and close up view of the repetitive segregated unit corresponding to the various types of GBs (LAGB, $\left.10.5^{\circ}\right), \sum 31 \mathrm{a}\left(17^{\circ}\right)$, $\sum 21 \mathrm{a}\left(22.5^{\circ}\right), \sum 13 \mathrm{~b}\left(25.5^{\circ}\right.$ and $\left.29^{\circ}\right), \sum 7\left(37.5^{\circ}\right)$ and random high angle GBs $\left(34^{\circ}\right.$ and $\left.43^{\circ}\right)$ are illustrated in Figs. 6 and 7 respectively and other GBs are provided in supporting information.

For LAGBs (Fig. 6(a) and Figure S2(supporting information)), it can be seen in the HAADF images that the $\mathrm{Cu}$ shows point segregation behavior. Highly segregated column (marked by the red ring) is surrounded by other low segregated columns. This type of behavior was prevailed for other GBs as well, until the misorien-

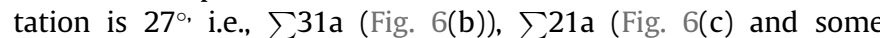
$\sum 13$ b GB (Fig. 6(d).

Point segregation is more evident at $\sum 13 \mathrm{~b}$ GB (Figs. 4(a) and $6(d))$, where the neighboring grains are misorientated by $27^{\circ}$ and $25.5^{\circ}$ respectively. However, when the misorientation is $29^{\circ}$, and beyond, parallel array segregation behavior can be seen for both CSL (Figs. 4(b) and 7(a,b) and S3,5 in the supporting information) and random high angle grain boundaries (r-HAGBs) (Fig. 7(c,d) and 

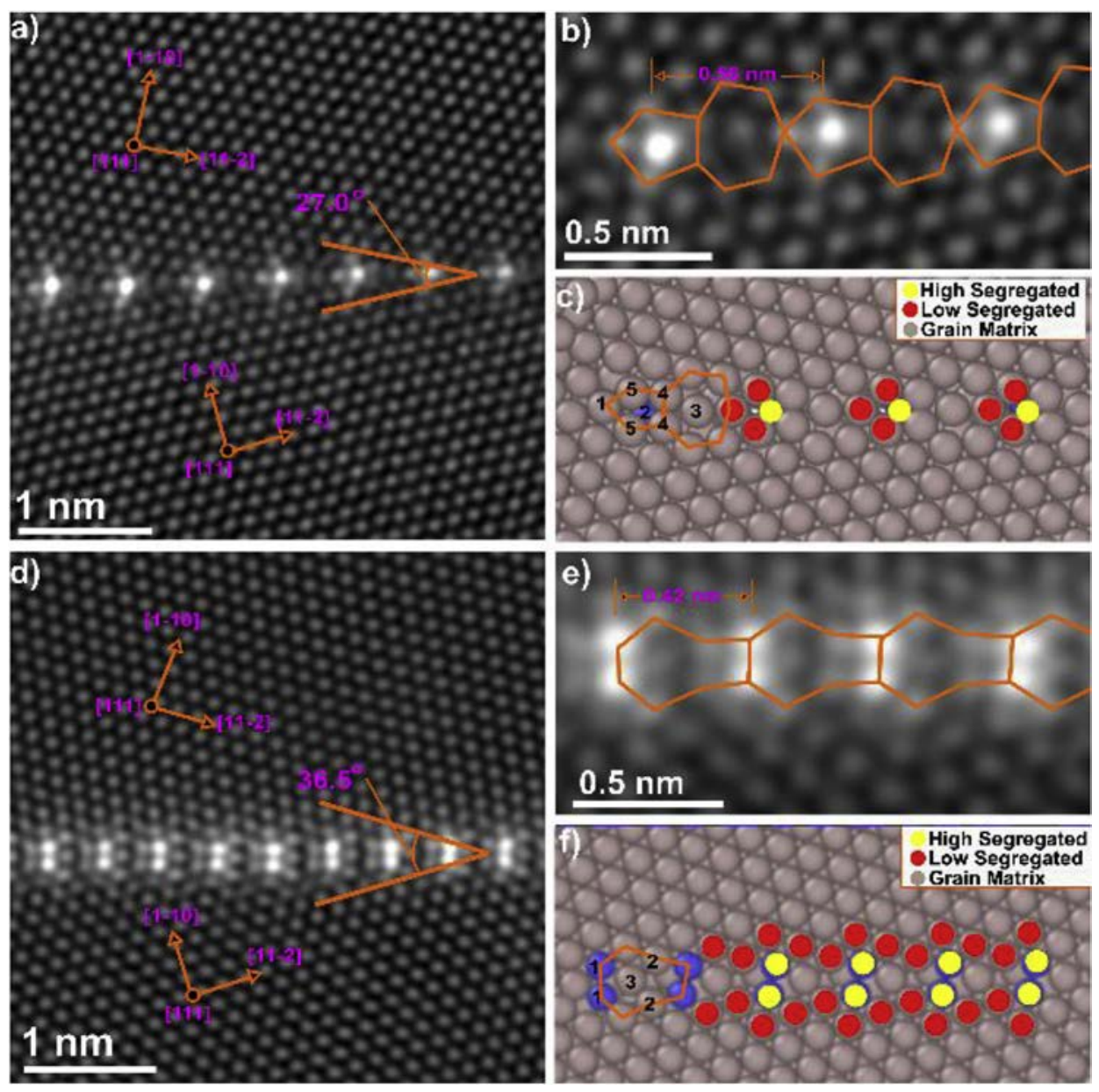

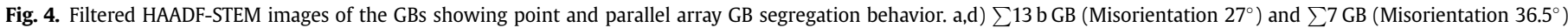

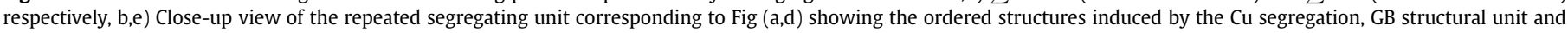

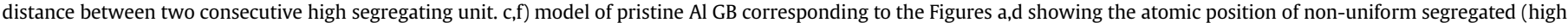
and low) columns.

a)

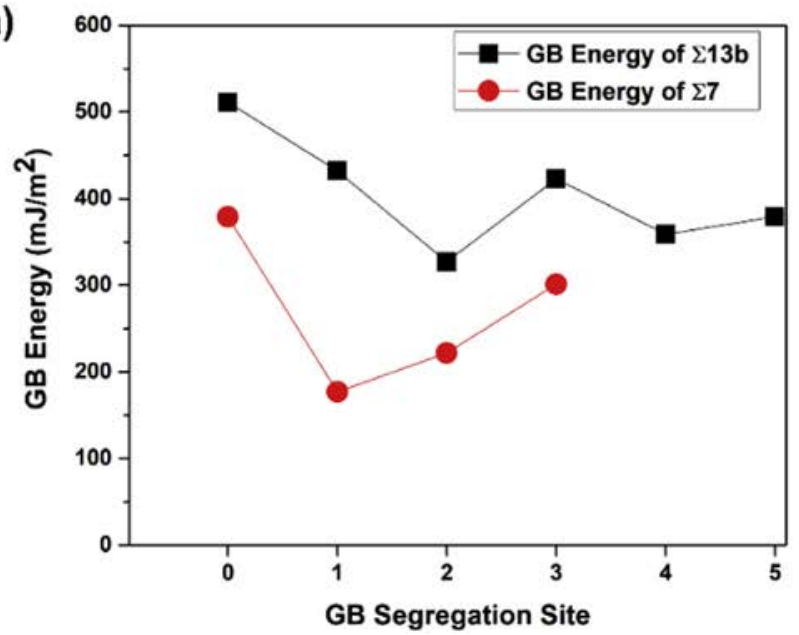

b)

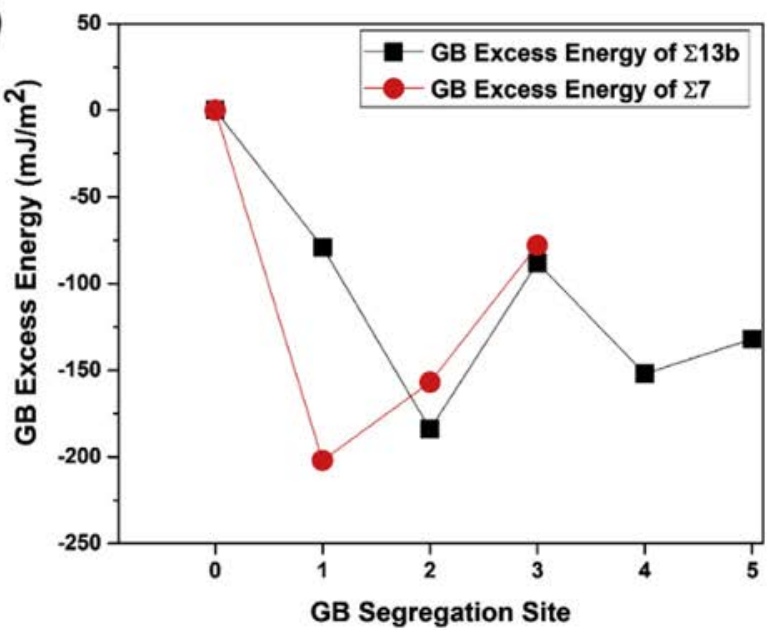

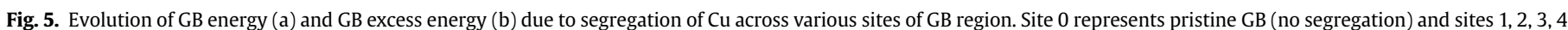
and 5 represents the various segregation sites shown in Fig. 4 (c,f).

S4). These results indicate that all the GBs of the films are segregated and by the varying unique nature of segregation. Results showed that GBs misorientated by less than $28^{\circ}$ demonstrated point segregation behavior whereas above $28^{\circ}$ demonstrated parallel array segregation behavior. We made an analysis of the spacing between two consecutive high segregated units for all the 

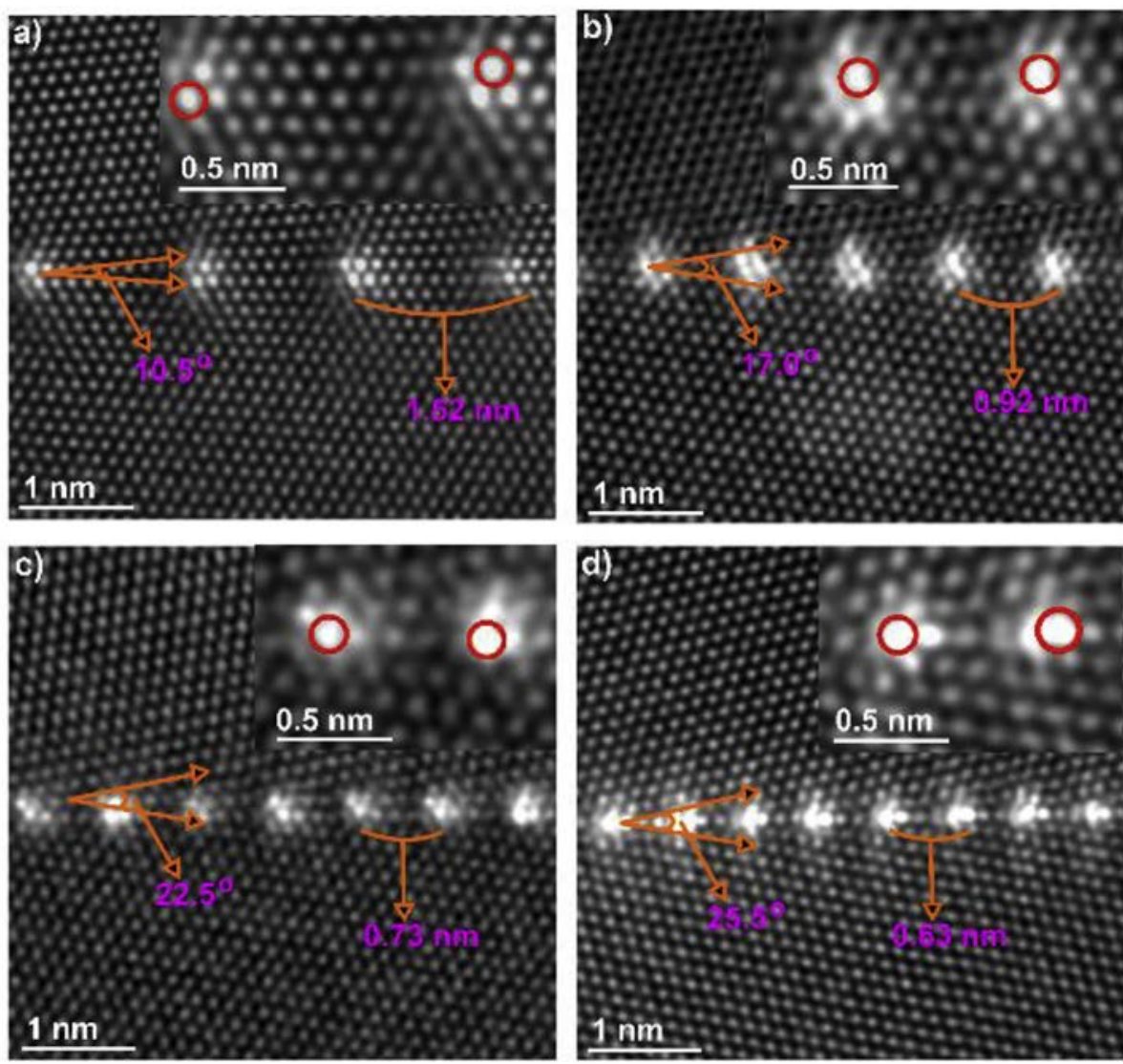

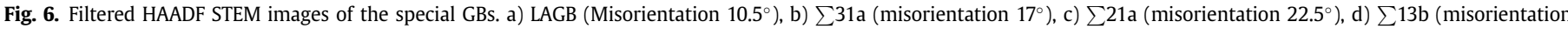

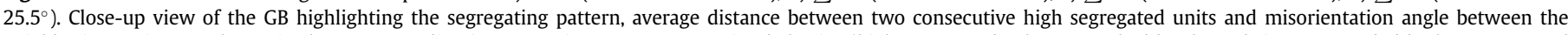

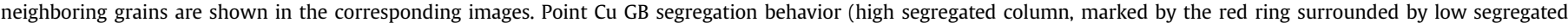
columns) is depicted at the boundary. (For interpretation of the references to color in this figure legend, the reader is referred to the Web version of this article.)

examined GBs. For LAGBs and majority of special GBs, the high segregated units are equidistant along the interface.

The plot of measured average inverse distances of two consecutive highly segregated units, as a function of misorientation angle is shown in Fig. 8. Only the distances corresponding to the GBs possessing equidistant segregating units are plotted in the figure. Angular range for different special GBs including LAGBs is marked by the rectangle of different colors (same colors used in GB contours). Furthermore, the linear plot of theoretical dislocation spacing is shown along with the experimental results for comparison. The theoretical dislocation spacing was calculated using dislocation model of LAGBs (spacings $(d=b / 2 \operatorname{Sin}(\alpha / 2) ; d$ is the dislocation spacing, $b$ is burger vector length and $\alpha$ is misorientation angle) [53]. It can be seen in the figure that the inverse distance increases with increase in misorientation. This increasing trend of inverse distance signifies that the segregation tendency of GBs increases with increase in misorientation. Contrast to LAGBs and majority of special GBs the high segregated units are not perfectly equidistant in r-HAGBs (Fig. 7(c,d) and S3) and some special GBs which are far from the ideal misorientation angle (Figure S3 and S5(a)). Among the r-HAGBs examined in this study, GBs misorientated by $31-34^{\circ}$, highly segregating units displayed transition spacing behavior between $\sum 13 \mathrm{~b}$ and $\sum 7$. Referring to Figs. $4(\mathrm{~d})$ and $7(a, b)$ and S5 it can be seen that the consecutive high segregated units are repeated after 5 to 4 atomic planes $(0.63 \mathrm{~nm}-0.56 \mathrm{~nm})$ in $\sum 13 \mathrm{~b}$ and 3 atomic planes $(0.42-0.39 \mathrm{~nm})$ in $\sum 7$. However, for $\mathrm{r}-$ HAGBs (Fig. 4(a)) misorientated by $34^{\circ}$, highly segregated columns are repeated by 3 and 2 atomic planes randomly. A similar pattern was observed in the r-HAGBs misorientated by $32^{\circ}$ (Supporting information Figure S4) as well.

Similarly, another r-HAGB misorientated by $42^{\circ}$ (Fig. 4(d)) shows highly segregated units repeated by 2 to 1 atomic planes. However, the r-HAGB misorientated by $43^{\circ}$ (supporting information S4(b)) shows a slightly different segregation pattern, segregating columns are repeated by two atomic planes three times followed by some gap and repeated. These results enabled us to conclude that the highly segregated units are equally spaced in LAGBs and majority of special GBs but not perfectly in r-HAGBs. However, the segregation tendency increases with increase in misorientation angle for all the GBs.

\section{Discussion}

It is now well established that certain alloying elements in multicomponent alloy preferentially segregate towards the GBs and they affect the materials properties profoundly [54,55]. Although the consequences of the phenomenon of GB segregation were observed more than 100 years ago and had been described theoretically and observed experimentally for decades, a vast majority of published reports were centralized for bcc iron considering ferritic steel as a basic construction material $[4,6]$. Only rarely such studies were done for other hosts even though, the application of aluminum or nickel base alloys has been rapidly increasing in the last decades. Particularly for Al alloys, the remarkable segregation of $\mathrm{Cu}$ and $\mathrm{Mg}$ solutes towards $\mathrm{Al} \mathrm{GBs,} \mathrm{and} \mathrm{their} \mathrm{effects} \mathrm{on} \mathrm{me-}$ chanical properties have been reported in a variety of experiments 

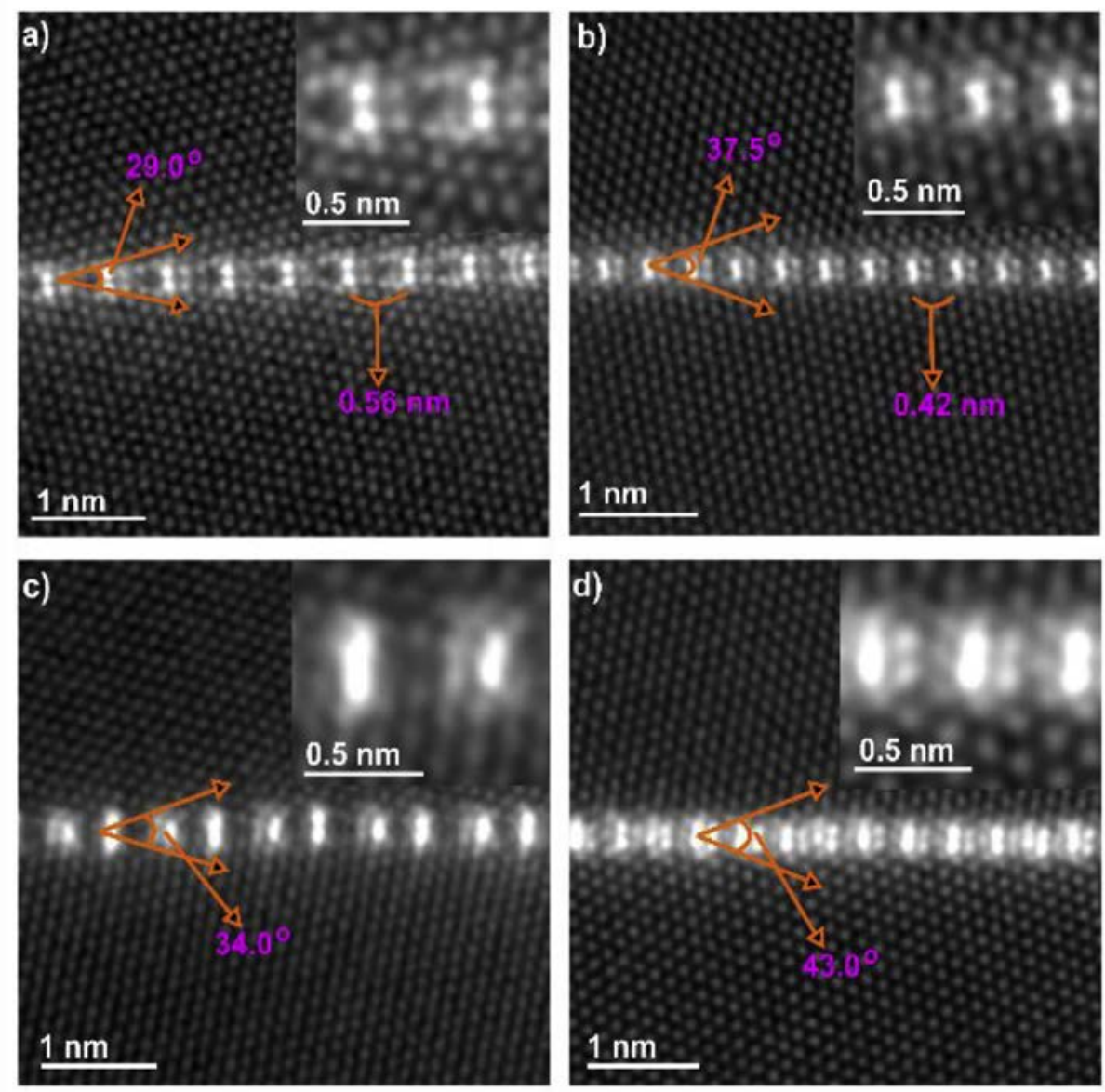

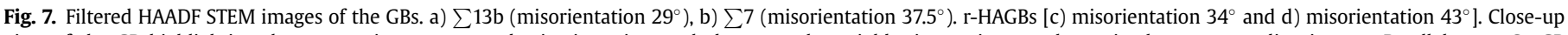

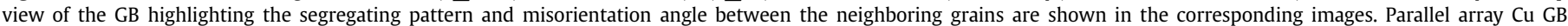
segregation behavior is depicted at the boundary.

[21,56-62]. However, apart from a very small number of atomicscale experimental studies $[21,63,64]$, the majority of the other studies were theoretical approaches. These studies are concentrated on segregation effects based on the substitutional core site segregation of $\mathrm{Mg}[56,57]$ and interstitial hollow site segregation [61,62] of $\mathrm{Cu}$, but little or no attention has been given to the experimental investigation of atomic scale adsorbate patterns, which impacts the important material's properties including but not limited to tensile strength, fatigue resistance, fracture toughness, strain hardening, brittleness, conductivity, or corrosion [1,6]. Here, we presented the detailed analysis of $\mathrm{Cu}$ segregation induced GB complexions at various GBs demonstrating unique segregation pattern for each type of GBs of the film.

Close observation of the graph of the average inverse distance of highly segregated units and theoretical dislocation spacing (Fig. 7) yielded two important conclusions. First, for LAGBs and CSL boundaries showing point segregation behavior, the two graphs (experimental and theoretical) coincide signifying the dislocations cores are highly segregated followed by low segregated neighboring columns showing point segregation behavior. This indicates that the segregation tendency is profoundly dominated by misorientation between two neighboring grains for LAGBs. A similar conclusion has been reported in an APT study of carbon segregation on ferrite GBs [64]. Second, for special and random HAGBs, a slight deviation of the inverse distances signifies that in addition to the misorientation other factors including GB geometry, boundary planes and orientations of each grain influenced the segregation behavior. However, it can be seen that the distance between two highly segregated columns approximately follow the theoretical dislocation spacing (increases with increase in misorientation) which enabled to infer that misorientation remains the dominating parameter even for HAGBs as well.

Furthermore, the trend of decrease in the distance of highly segregated units with an increase in misorientation suggested that the segregation tendency of GBs increases with increases in misorientation between the neighboring grains [31,64]. Some contrasting results on segregation at special GBs are reported, for example, the APT study of carbon segregation to ferrite GBs showed too low segregation at the ideally misorientated CSL boundaries [64] whereas Ag segregation at Cu GBs showed stronger segregation of Ag at $\sum 5$ (310) GBs than r-HAGBs [65]. Our study shows that segregation tendency is misorientation dependence. Within the angular regime of corresponding special GB ( $\sum 7$ and $\left.\sum 13 b\right)$, it followed the trend of misorientation dependence, i.e., the distance between two highly segregated units decreased with increase in misorientation from $25.5^{\circ}$ to $30^{\circ}\left(\sum 13 \mathrm{~b}\right)$ and $36^{\circ}-40^{\circ}\left(\sum 7\right)$. This slight differences in the spacing of high segregated units among the same special GB class could be attributed to the impact of GB planes and the structurally different secondary dislocations of the GB on segregation behavior [64]. Referring to the five macroscopic degree of GB character, only misorientation dependence of GB complexions are explored in this study. Since, the film is strongly $<111>$ textured, all the grains are orientated along $<111>$ direction, and the rotation axis, another parameter of five macroscopic degrees of 


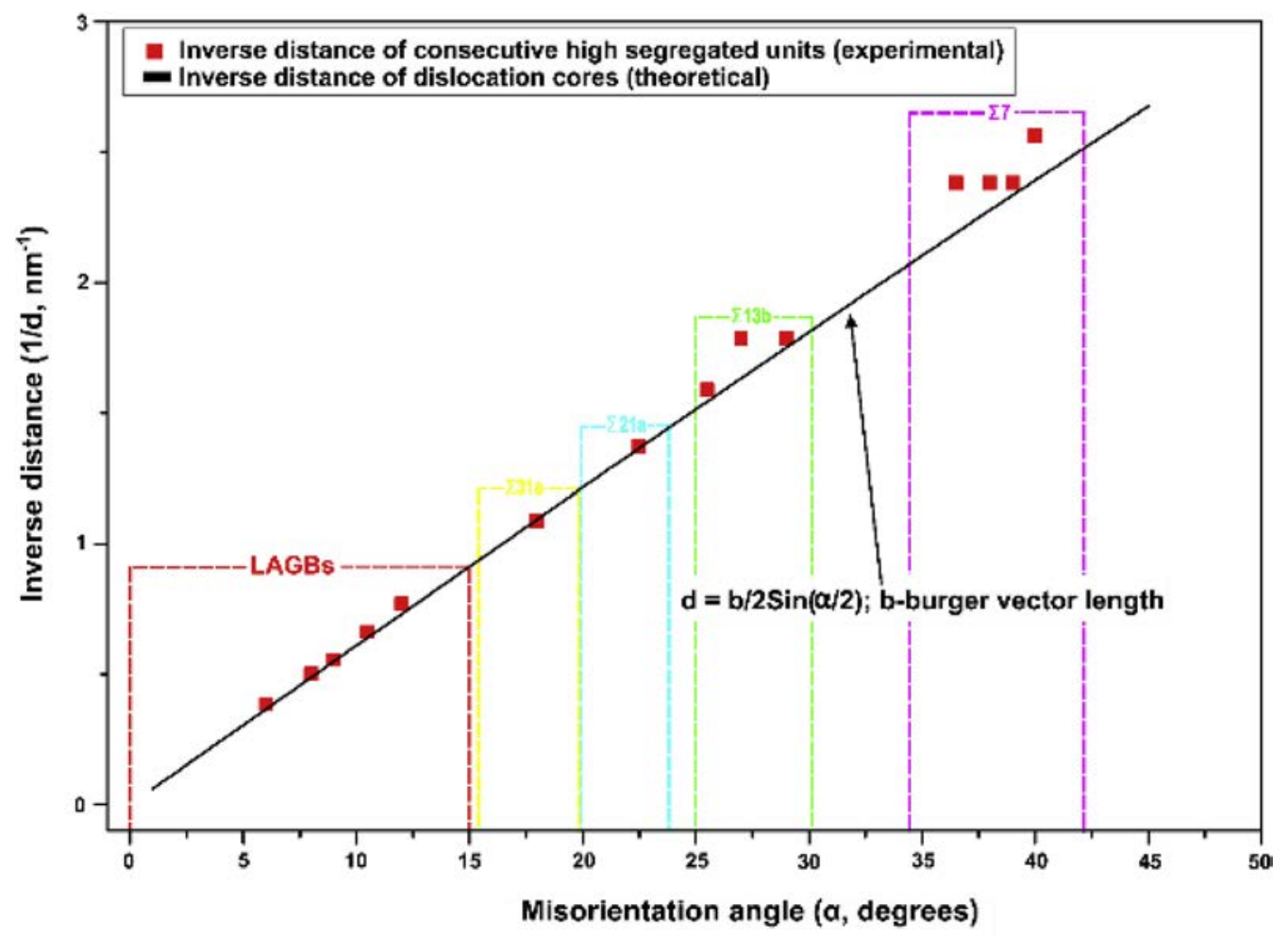

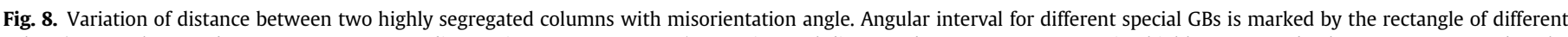

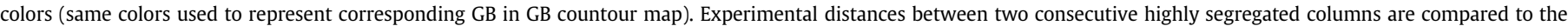

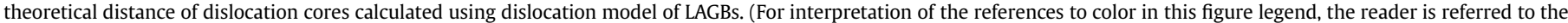
Web version of this article.)

freedom of GB character is $<111>$ for all the GBs. Similarly, all the GBs are symmetric tilt GBs, as a consequence, all the grain boundary plane normals are similar or close to (110). Hence, although grain boundary normal and grain orientation i.e. rotation axes significantly influences grain boundary energy, stability and consequently the GB segregation behavior and pattern [66-68], these parameters do not significantly impacted the core ideas in this particular analysis and are not explored in the current study.

Regarding segregation behavior the majority of the studies demonstrated monolayer point segregation behavior in highresolution electron microscopy study of thin films. Some multilayer GB complexions were reported at high temperature across HAGBs [23-27]. These multilayer GB complexions in HAGBs of $\mathrm{Cu}$ alloy systems are referred as the consequences of temperature induced GB complexions transformation [27,28]. In this study, we demonstrated point segregation behavior for GBs misorientated by less than $28^{\circ}$, and parallel array segregation behavior for GBs misorientated by more than $28^{\circ}$. Since only HAGBs showed parallel array segregation behavior, we could predict that these parallel array multilayer GB complexions with parallel array segregation behavior could be the results of the temperature-induced point segregated GB complexions transformation. However, this remains a speculation unless extensive experimental and theoretical works provide the necessary evidences.

Numerous prior experimental and theoretical works on the segregation tendency of $\mathrm{Cu}$ and $\mathrm{Mg}$ towards $\mathrm{Al}$ GBs [56,57,59,69-72] and segregation effects (cohesion enhancing effect of $\mathrm{Mg}$ [58,59,73-77] (with some controversies [58,73])), and the cohesion effect of $\mathrm{Cu}$ (less comprehensive studies) [60] were based on point segregation behavior. It has been illustrated that the strengthening effect of $\mathrm{Cu}$ solutes lies in the creation of new $\mathrm{Cu}-\mathrm{Al}$ bonds across the grain boundary, thereby increasing its resistance against intergranular cleavage [60]. Herein, we provided experimental evidence that different types of GBs are segregated uniquely from point to parallel array Cu GB segregation behavior presenting extensive atomic scale analysis on $\mathrm{Cu}$-adsorbate arrangement at GBs. This novel finding, parallel array GB segregation pattern definitely influences the existing understanding of $\mathrm{Cu}$ GB cohesion phenomena (based on the point segregation behavior) and consequently the mechanical performance of the material. Our future works are directed towards this aspect of analysis, and also the detailed density functional theory to further explore the thermodynamic and geometrical factors governing these two types of segregation behavior and their implications on mechanical along with electronic, magnetic, and diffusion-related properties of the films, as a consequence of this $\mathrm{Cu}$ segregation behavior.

\subsection{The role of the displacement field in GB segregation}

It must be noted that a coincidence sites interface occurs for angles whose tangent is a rational number. Given that rational numbers are a dense set, it turns out that, all interfaces are, in practical terms coincidence interfaces. The value quoted for $\Sigma$ is there for description purposes only and is obtained by using the Ranganathan formula applied to the ratio of integers obtained from a continued fraction expansion of the angle to an accuracy of $0.01^{\circ}$. In view of the obvious limitations of the CSL theory to describe general interfaces, it turns out that the strain field, which is smaller for lower values of $\Sigma$ is a more appropriate concept in the study of geometrical properties of interfaces.

In this section we shall show that the two types of segregation observed, namely point and parallel array as well as the variation of 
the distance between highest segregation spots can be explained by means of a displacement field obtained from the dichromatic pattern as described in Ref. [73]. Using Bollmann's ideas with the added definition of singularity given by equation (1) and assuming that segregation takes place preferentially at sites where the strain field is larger, it is possible to account not only for the two types of segregation observed here i.e., point and parallel array, but also for the variation of the distance between segregation spot patterns due to the presence of secondary dislocations. Given that an interface is a system that exists on the surface between two differently oriented crystals when they are brought into contact, the best way to describe its geometry is by using a property that depends only on this surface and not on the final atomic positions near the relaxed boundary which depend on the specifics of the interatomic potential. One such property is the displacement field as given by the set of vectors smaller than one atomic diameter that join differently colored sites in a dichromatic pattern that would result in electron cloud overlap if replaced by actual atoms. This field is illustrated in Fig. 9(a) which shows the displacement field of a twist interface between two hexagonal planes rotated by 16.43 .degrees. The small blue and red circles in the figure represent the sites of each lattice while the thick black lines joining their canters represent the displacement vectors $d_{i}$ of the field. The large grey circles are centred on the middle of each displacement vector $\bar{x}_{i}$ with a diameter of one interatomic distance serve as a visual aid to identify the symmetry properties of the field $\left\{\bar{x}_{i}, d_{i}\right\}$ which varies with the rotation angle/axis pair and can be used to characterize the interface. In the figure, the filled blue circles represent the O-lattice sites where the strain field is zero and the blue hexagons represent the network of dislocation lines.

In order to build the strain field of the tilt interfaces studied in this work, where the rotation axis is parallel to the interfacial plane, the strain field must also be calculated along the $\mathrm{z}$ axis. This entails repeating the calculation shown in Fig. 9(a) for all distinct crystal planes along the $z$ direction. Since the stacking of (111) planes in FCC crystals is A-B-C, we need to consider the strain field in each A, $B$ and $C$ plane along the $<111>$ rotation axis. The result of doing this is shown in Fig. 9(b) which, using smaller red, green and blue circles shows the displacement fields all planes allowing them to be simultaneously displayed in a single $2 \mathrm{D}$ diagram. A tilt boundary is then recreated by choosing an interfacial plane (represented by the black line segment in the figure) and leaving the sites of each crystalline lattice on either side of the boundary. The interfacial plane is normally chosen to run along a direction that maximizes the density of O-points which being sites of minimum strain, should minimize the interfacial energy. According to Bollmann [74], there are special i.e., singular low energy interfaces which contain only primary dislocations with crystalline Burgers vectors. Under small angular deviations $\Delta \theta$ from a singular orientation the interface preserves the singular configuration by concentrating the displacements along secondary dislocations with non-crystalline Burgers vectors belonging to the DSC lattice. Bollmann did not have a rigorous definition of singularity so he could derive analytical formulas for the location of secondary dislocations, this limitation can be overcome by using the definition of singularity given in Ref. [75]. Accordingly, singular interfaces between rotated lattices are those whose rotation angle $\theta$ is given by

$$
\theta=2 \tan ^{-1}(\sqrt{N} / n)
$$

where $N=\sqrt{h^{2}+k^{2}+l^{2}}$ is the magnitude of the rotation axis $<h, k, l>$ and $\mathrm{n}$ is an integer. Since secondary dislocation spacing varies with $\Delta \theta$, it may vary along the interface as a consequence of small variations in $\Delta \theta$ near the interfacial plane arising from localized elastic strains as exemplified by the lattice distortion observed near the interface of Fig. 7(a). Fig. 9(b) shows the 3D strain field of the singular $\Sigma 19, \theta=10.4^{\circ}$ interface corresponding to $n=19$ in equation, which is an example of point segregation, while Fig. 10 shows the strain fields of two secondary interfaces showing parallel array segregation. Note the blue lines in the inset of Fig. 10(d) mark the positions of secondary dislocations showing how these are responsible for the change in the segregation pattern made evident by the encircled brightest spots.
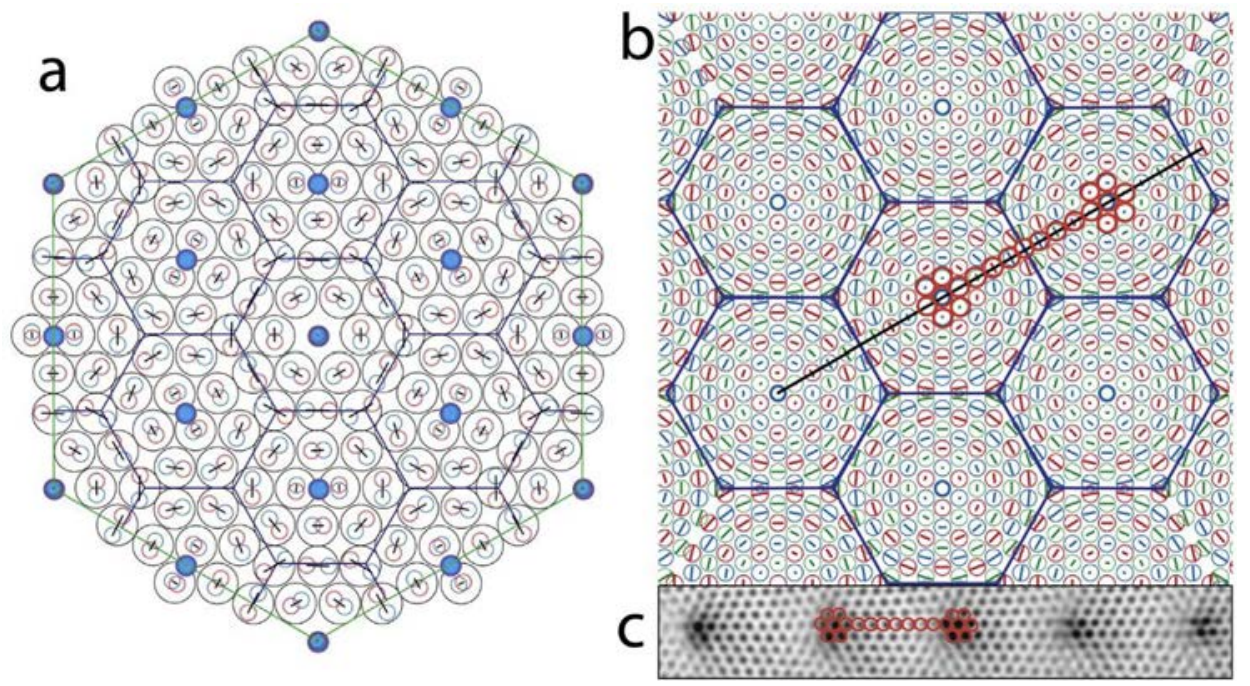

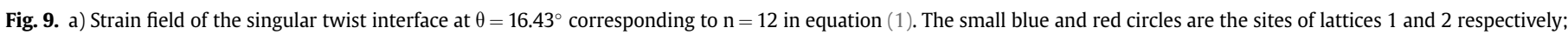

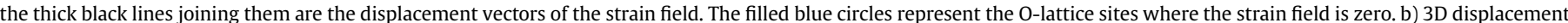

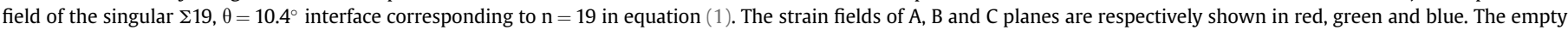

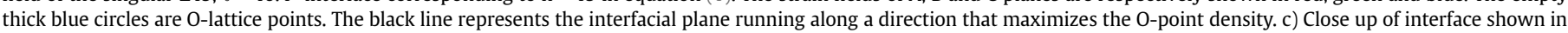

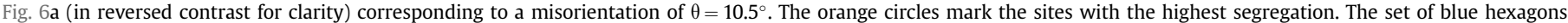

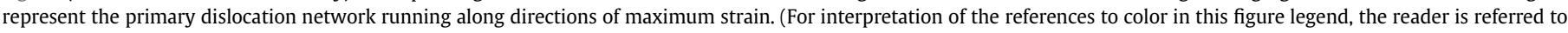
the Web version of this article.) 

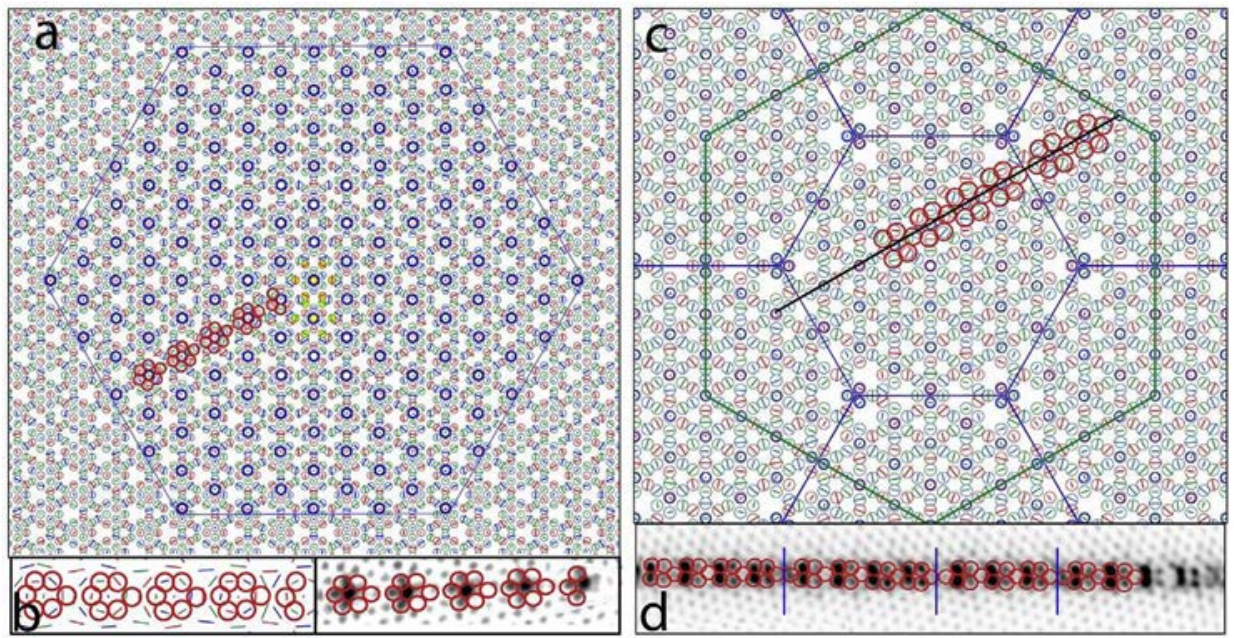

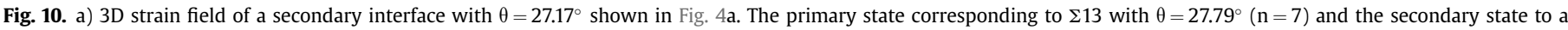

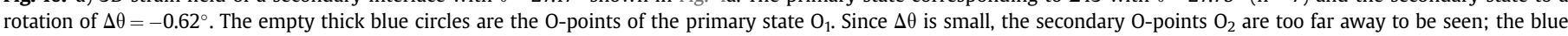

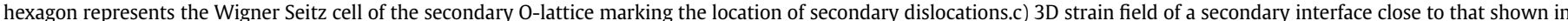

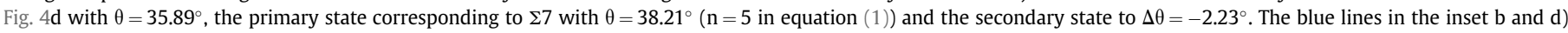

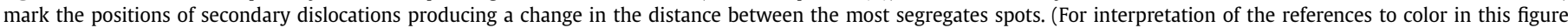
legend, the reader is referred to the Web version of this article.)

\section{Conclusions}

A detailed systematic study was conducted to investigate the misorientation dependence on the structure and chemistry of segregation induced GB complexions of Al 7075 alloy GBs. We found that $\mathrm{Cu}$ as a principle $\mathrm{GB}$ segregating entities of this alloy gets accumulated on every GBs (LAGBs, special GBs, and r-HAGBs) with varying unique segregation pattern. Multilayer (extended up to 4 atomic planes) non-uniformly (low and high) segregated mixed atomic columns showing two types of Cu GB segregation behavior namely, point and parallel array were observed at the GBs which has been explained in terms of the displacement field. Results showed that $\mathrm{Cu}$ segregation behavior greatly depends on the misorientation angle between two neighboring grains for $<111>$ symmetric tilt GBs of strong $<111>$ textured film. GBs misorientated by less than $28^{\circ}$ displayed point (high segregated atomic column surrounded by low segregated columns) segregation behavior whereas more than the $28^{\circ}$ displayed parallel array (two highly segregated columns opposite to each other across the interface surrounded by low segregated columns) segregation behavior. GB complexions with parallel array segregation behavior are inferred as a result of the temperature induced point segregated GB complexions transformation. For point segregated GBs (misorientation $<28^{\circ}$ ), atomic column at the dislocation core is highly segregated. Furthermore, based on the distance between two high segregated units, we concluded that highly misorientated GBs are more segregated than low misoriented GBs. The detailed analysis of the atomic scale arrangements of $\mathrm{Cu}$ adsorbate on the commercially used $\mathrm{Al}$ alloy GBs is presented. We believe this work is an important contribution in the field of GB segregation engineering and potentially help in the better understanding of the implications of GB segregation on materials performance.

\section{Acknowledgments}

The authors are thankful to the Welch Foundation Grant (No. AX-1615) and the Department of Defense grant \#W911NF-18-10439. We thank to Dr. Josefina Arellano-Jimenez for her technical support in the Kleberg Advanced Microscopy Center at UTSA.

\section{Appendix A. Supplementary data}

Supplementary data to this article can be found online at https://doi.org/10.1016/j.actamat.2019.09.010.

\section{References}

[1] A.P. Sutton, R.W. Balluffi, Interfaces in Crystalline Materials, 1995.

[2] D. Raabe, M. Herbig, S. Sandlöbes, Y. Li, D. Tytko, M. Kuzmina, D. Ponge, P.P. Choi, Grain boundary segregation engineering in metallic alloys: a pathway to the design of interfaces, Curr. Opin. Solid State Mater. Sci. 18 (4) (2014) 253-261.

[3] W. Kingery, Plausible concepts necessary and sufficient for interpretation of ceramic grain-boundary phenomena: I. Grain-Boundary characteristics, structure, and electrostatic potential, J. Am. Ceram. Soc. 57 (1) (1974) 1-8.

[4] P. Lejček, M. Šob, V. Paidar, Interfacial segregation and grain boundary embrittlement: an overview and critical assessment of experimental data and calculated results, Prog. Mater. Sci. 87 (2017) 83-139.

[5] M. Seah, E. Hondros, Grain boundary segregation, Proc. R. Soc. London, Ser. A 335 (1601) (1973) 191-212.

[6] P. Lejcek, Grain Boundary Segregation in Metals, Springer Science \& Business Media, 2010.

[7] T. Watanabe, Grain boundary engineering: historical perspective and future prospects, J. Mater. Sci. 46 (12) (2011) 4095-4115.

[8] P.R. Cantwell, M. Tang, S.J. Dillon, J. Luo, G.S. Rohrer, M.P. Harmer, Grain boundary complexions, Acta Mater. 62 (2014) 1-48.

[9] D. Raabe, S. Sandlöbes, J. Millán, D. Ponge, H. Assadi, M. Herbig, P.-P. Choi, Segregation engineering enables nanoscale martensite to austenite phase transformation at grain boundaries: a pathway to ductile martensite, Acta Mater. 61 (16) (2013) 6132-6152.

[10] G.S. Rohrer, D.M. Saylor, B.E. Dasher, B.L. Adams, A.D. Rollett, P. Wynblatt, The distribution of internal interfaces in polycrystals, Z. Met. 95 (4) (2004) 197-214.

[11] G.S. Rohrer, Grain boundary energy anisotropy: a review, J. Mater. Sci. 46 (18) (2011) 5881.

[12] P. Lejček, On the anisotropy of grain boundary segregation in multicomponent systems, Prog. Surf. Sci. 35 (1-4) (1990) 209-213.

[13] J.P. Buban, K. Matsunaga, J. Chen, N. Shibata, W.Y. Ching, T. Yamamoto Y. Ikuhara, Grain boundary strengthening in alumina by rare earth impurities, Science 311 (5758) (2006) 212-215.

[14] R. Klie, N. Browning, Atomic scale characterization of oxygen vacancy segregation at SrTiO 3 grain boundaries, MRS Online Proc. Libr. Arch.. 654 (2000).

[15] N. Shibata, S.J. Pennycook, T.R. Gosnell, G.S. Painter, W.A. Shelton, P.F. Becher Observation of rare-earth segregation in silicon nitride ceramics at subnanometre dimensions, Nature 428 (6984) (2004) 730-733.

[16] A. Ziegler, J.C. Idrobo, M.K. Cinibulk, C. Kisielowski, N.D. Browning R.O. Ritchie, Interface structure and atomic bonding characteristics in silicon nitride ceramics, Science 306 (5702) (2004) 1768-1770.

[17] G.B. Winkelman, C. Dwyer, T.S. Hudson, D. Nguyen-Manh, M. Döblinger, 
R.L. Satet, M.J. Hoffmann, D.J. Cockayne, Three-dimensional organization of rare-earth atoms at grain boundaries in silicon nitride, Appl. Phys. Lett. 87 (6) (2005), 061911.

[18] G. Duscher, M.F. Chisholm, U. Alber, M. Ruhle, Bismuth-induced embrittlement of copper grain boundaries, Nat. Mater. 3 (9) (2004) 621-626.

[19] U. Alber, H. Müllejans, M. Rühle, Bismuth segregation at copper grain boundaries, Acta Mater. 47 (15-16) (1999) 4047-4060.

[20] Z. Wang, M. Saito, K.P. McKenna, L. Gu, S. Tsukimoto, A.L. Shluger, Y. Ikuhara, Atom-resolved imaging of ordered defect superstructures at individual grain boundaries, Nature 479 (7373) (2011) 380-383.

[21] P. Parajuli, R. Mendoza-Cruz, A. Hurtado-Macias, U. Santiago, M.J. Yacamán, A direct observation of ordered structures induced by $\mathrm{Cu}$ segregation at grain boundaries of Al 7075 alloys, Phys. Status Solidi 215 (17) (2018) 1-8.

[22] Z. Yu, P.R. Cantwell, Q. Gao, D. Yin, Y. Zhang, N. Zhou, G.S. Rohrer, M. Widom, J. Luo, M.P. Harmer, Segregation-induced ordered superstructures at general grain boundaries in a nickel-bismuth alloy, Science 358 (6359) (2017) 97-101.

[23] T. Frolov, S.V. Divinski, M. Asta, Y. Mishin, Effect of interface phase transformations on diffusion and segregation in high-angle grain boundaries, Phys Rev. Lett. 110 (25) (2013) 255502.

[24] J. Luo, H. Cheng, K.M. Asl, C.J. Kiely, M.P. Harmer, The role of a bilayer interfacial phase on liquid metal embrittlement, Science 333 (6050) (2011) $1730-1733$.

[25] A. Kundu, K.M. Asl, J. Luo, M.P. Harmer, Identification of a bilayer grain boundary complexion in Bi-doped Cu, Scr. Mater. 68 (2) (2013) 146-149.

[26] S.J. Dillon, M. Tang, W.C. Carter, M.P. Harmer, Complexion: a new concept for kinetic engineering in materials science, Acta Mater. 55 (18) (2007) 6208-6218

[27] T. Frolov, M. Asta, Y. Mishin, Segregation-induced phase transformations in grain boundaries, Phys. Rev. B 92 (2) (2015), 020103.

[28] T. Frolov, D.L. Olmsted, M. Asta, Y. Mishin, Structural phase transformations in metallic grain boundaries, Nat. Commun. 4 (2013) 1899

[29] A.P. Sutton, R.W. Balluffi, Interfaces in Crystalline Materials, 1995.

[30] V. Randle, The coincidence site lattice and the 'sigma enigma', Mater. Char. 47 (5) (2001) 411-416.

[31] P. Parajuli, R. Mendoza-Cruz, U. Santiago, A. Ponce, M.J. Yacamán, The evolution of growth, crystal orientation, and grain boundaries disorientation distribution in gold thin films, Cryst. Res. Technol. 53 (8) (2018) 1-7.

[32] P. Parajuli, R. Mendoza-Cruz, J.J. Velazquez-Salazar, M. Jose Yacaman, A. Ponce, Fivefold annealing twin in nanocrystalline Au/Pd film, Mater. Lett. 244 (2019) 88-91.

[33] E.F. Rauch, M. Véron, Automated crystal orientation and phase mapping in TEM, Mater. Char. 98 (2014) 1-9.

[34] S. Plimpton, Fast parallel algorithms for short-range molecular dynamics, J. Comput. Phys. 117 (1) (1995) 1-19.

[35] M. Mendelev, M. Kramer, C.A. Becker, M. Asta, Analysis of semi-empirical interatomic potentials appropriate for simulation of crystalline and liquid A and Cu, Philos. Mag. 88 (12) (2008) 1723-1750.

[36] J. Cai, Y. Ye, Simple analytical embedded-atom-potential model including a long-range force for fcc metals and their alloys, Phys. Rev. B 54 (12) (1996) 8398.

[37] M. Mendelev, D. Srolovitz, G. Ackland, S. Han, Effect of Fe segregation on the migration of a non-symmetric $\Sigma 5$ tilt grain boundary in Al, J. Mater. Res. 20 (1) (2005) 208-218.

[38] M. Tschopp, D. McDowell, Structures and energies of $\Sigma 3$ asymmetric tilt grain boundaries in copper and aluminium, Philos. Mag. 87 (22) (2007) 3147-3173.

[39] J. Rittner, D. Seidman, $<110>$ symmetric tilt grain-boundary structures in fcc metals with low stacking-fault energies, Phys, Rev. B 54 (10) (1996) 6999.

[40] L. Zhang, C. Lu, K. Tieu, Molecular dynamics simulation on $\Sigma 5$ grain boundaries of copper bicrystal under tensile and shear deformation, MRS Online Proc. Libr. Arch. 1651 (2014)

[41] A. Stukowski, Visualization and analysis of atomistic simulation data with OVITO-the open visualization tool, Model. Simul. Mater. Sci. Eng. 18 (1) (2009), 015012.

42] P. Parajuli, R. Mendoza-Cruz, M.J. Yacamán, A. Ponce, Alloying and Annealing Effects on Grain Boundary Character Evolution of Al-Alloy 7075 Thin Films: an ACOM-TEM Analysis, Springer International Publishing, Cham, 2019, pp. 109-119.

[43] M. Déchamps, F. Baribier, A. Marrouche, Grain-boundaries: criteria of specialness and deviation from CSL misorientation, Acta Metall. 35 (1) (1987) 101-107.

[44] Y. Zhou, U. Erb, K. Aust, G. Palumbo, The effects of triple junctions and grain boundaries on hardness and Young's modulus in nanostructured $\mathrm{Ni}-\mathrm{P}$, Scr. Mater. 48 (6) (2003) 825-830.

[45] V.V. Singh, A.H. King, G. Dixit, Some further microstructural characteristics of face-centered cubic polycrystalline metal thin films, J. Electron. Mater. 26 (9) (1997) 987-995.

[46] I. Dillamore, R. Smallman, The stacking-fault energy of FCC metals, Philos. Mag. 12 (115) (1965) 191-193.

[47] F.J. Humphreys, M. Hatherly, Recrystallization and Related Annealing Phenomena, Elsevier, 2012

[48] S. Kobayashi, H. Takagi, T. Watanabe, Grain boundary character distribution and texture evolution during surface energy-driven grain growth in nanocrystalline gold thin films, Philos. Mag. 93 (10-12) (2013) 1425-1442.

[49] S. Kim, Y. Jung, J.J. Kim, S. Lee, H. Lee, Z-contrast dependence of quantitative scanning transmission electron microscopy image of Si1 - xGex binary crystals, J. Alloy. Comp. 618 (2015) 545-550.

[50] G. Martinez, A. Rosenauer, A. De Backer, J. Verbeeck, S. Van Aert, Quantitative composition determination at the atomic level using model-based high-angle annular dark field scanning transmission electron microscopy, Ultramicroscopy 137 (2014) 12-19.

[51] V. Grillo, The effect of surface strain relaxation on HAADF imaging, Ultramicroscopy 109 (12) (2009) 1453-1464.

[52] M. Tschopp, D. McDowell, Asymmetric tilt grain boundary structure and energy in copper and aluminium, Philos. Mag. 87 (25) (2007) 3871-3892.

[53] W.T. Read, W. Shockley, Dislocation models of crystal grain boundaries, Phys. Rev. 78 (3) (1950) 275.

[54] P. Lejček, S. Hofmann, Thermodynamics and structural aspects of grain boundary segregation, Crit. Rev. Solid State 20 (1) (1995) 1-85.

[55] M.J. Duarte, J. Klemm, S.O. Klemm, K.J. Mayrhofer, M. Stratmann, S. Borodin, A.H. Romero, M. Madinehei, D. Crespo, J. Serrano, S.S. Gerstl, P.P. Choi, D. Raabe, F.U. Renner, Element-resolved corrosion analysis of stainless-type glass-forming steels, Science 341 (6144) (2013) 372-376.

[56] D.R. Baer, C. Windisch Jr., M.H. Engelhard, M.J. Danielson, R.H. Jones, J.S. Vetrano, Influence of Mg on the corrosion of Al, J. Vac. Sci. Technol. A 18 (1) (2000) 131-136.

[57] J. Chen, T. Sun, R. Viswanadham, J. Green, Grain boundary segregation of an Al-Zn-Mg ternary alloy, Metall. Trans. A 8 (12) (1977) 1935-1940.

[58] R. Song, M. Tseng, B. Zhang, J. Liu, Z. Jin, K. Shin, Grain boundary segregation and hydrogen-induced fracture in 7050 aluminium alloy, Acta Mater. 44 (8) (1996) 3241-3248.

[59] G. Sha, L. Yao, X. Liao, S.P. Ringer, Z.C. Duan, T.G. Langdon, Segregation of solute elements at grain boundaries in an ultrafine grained $\mathrm{Al}-\mathrm{Zn}-\mathrm{Mg}-\mathrm{Cu}$ alloy, Ultramicroscopy 111 (6) (2011) 500-505.

[60] D. Zhao, O.M. Løvvik, K. Marthinsen, Y. Li, Segregation of Mg, Cu and their effects on the strength of Al $\Sigma 5$ (210)[001] symmetrical tilt grain boundary, Acta Mater. 145 (2018) 235-246.

[61] X.-Y. Liu, C.-L. Liu, L. Borucki, A new investigation of copper's role in enhancing $\mathrm{Al}-\mathrm{Cu}$ interconnect electromigration resistance from an atomistic view, Acta Mater. 47 (11) (1999) 3227-3231.

[62] G.H. Campbell, J.M. Plitzko, W.E. King, S.M. Foiles, C. Kisielowski, G.J. Duscher, Copper segregation to the $\Sigma 5(310) /[001]$ symmetric tilt grain boundary in aluminum, Interface Sci. 12 (2) (2004) 165-174.

[63] S. Schmidt, W. Sigle, W. Gust, M. Rühle, Gallium segregation at grain boundaries in aluminium, Z. Met. 93 (5) (2002) 428-431.

[64] M. Herbig, D. Raabe, YJ. Li, P. Choi, S. Zaefferer, S. Goto, Atomic-scale quantification of grain boundary segregation in nanocrystalline material, Phys. Rev. Lett. 112 (12) (2014) 126103.

[65] S.V. Divinski, H. Edelhoff, S. Prokofjev, Diffusion and segregation of silver in copper $\Sigma 5$ (310) grain boundary, Phys. Rev. B 85 (14) (2012) 144104.

[66] C.M. Barr, G.A. Vetterick, K.A. Unocic, K. Hattar, X.-M. Bai, M.L. Taheri, Anisotropic radiation-induced segregation in 316L austenitic stainless steel with grain boundary character, Acta Mater. 67 (2014) 145-155.

[67] K. Matsunaga, H. Nishimura, S. Hanyu, H. Muto, T. Yamamoto, Y. Ikuhara, HRTEM study on grain boundary atomic structures related to the sliding behavior in alumina bicrystals, Appl. Surf. Sci. 241 (1-2) (2005) 75-79.

[68] D.S. D'Antuono, J. Gaies, W. Golumbfskie, M. Taheri, Grain boundary misorientation dependence of $\beta$ phase precipitation in an $\mathrm{Al}-\mathrm{Mg}$ alloy, Scr. Mater. 76 (2014) 81-84.

[69] J. Pickens, T. Langan, The effect of solution heat-treatment on grain boundary segregation and stress-corrosion cracking of Al-Zn-Mg alloys, Metall. Trans. A 18 (10) (1987) 1735-1744.

[70] P. Doig, J.W. Edington, The influence of quenching on the segregation of magnesium to the grain boundaries in an Al-5.9 wt pct Zn-3.2 wt pct Mg alloy, Metall. Mater. Trans. A 6 (4) (1975) 943-945.

[71] R. Jones, D. Baer, M. Danielson, J. Vetrano, Role of Mg in the stress corrosion cracking of an Al-Mg alloy, Metall. Mater. Trans. A 32 (7) (2001) 1699-1711.

[72] T. Malis, M.C. Chaturvedi, Grain-boundary segregation in an Al-8 wt \% Mg alloy, J. Mater. Sci. 17 (5) (1982) 1479-1486.

[73] X.-Y. Liu, J. Adams, Grain-boundary segregation in $\mathrm{Al}-10 \% \mathrm{Mg}$ alloys at hot working temperatures, Acta Mater. 46 (10) (1998) 3467-3476.

[74] R.G. Song, W. Dietzel, B.J. Zhang, W.J. Liu, M.K. Tseng, A. Atrens, Stress corrosion cracking and hydrogen embrittlement of an $\mathrm{Al}-\mathrm{Zn}-\mathrm{Mg}-\mathrm{Cu}$ alloy, Acta Mater. 52 (16) (2004) 4727-4743.

[75] L. Xiaoguang, W. Xiaowei, W. Jingyang, Z. Hongyan, First-principles investigation of $\mathrm{Mg}$ segregation at $\Sigma=11(113)$ grain boundaries in $\mathrm{Al}$, J. Phys. Condens. Matter 17 (27) (2005) 4301.

[76] S. Zhang O.Y. Kontsevoi, A.J. Freeman, G.B. Olson, Cohesion enhancing effect of magnesium in aluminum grain boundary: a first-principles determination, Appl. Phys. Lett. 100 (23) (2012) 231904

[77] V.I. Razumovskiy, A.V. Ruban, I.M. Razumovskii, A.Y. Lozovoi, V.N. Butrim, Y.K. Vekilov, The effect of alloying elements on grain boundary and bulk cohesion in aluminum alloys: an ab initio study, Scr. Mater. 65 (10) (2011) 926-929. 